\title{
Successful generation of cloned mice using nuclear transfer from induced pluripotent stem cells
}

Cell Research (2010) 20:850-853. doi:10.1038/cr.2010.78; published online 15 June 2010

\section{Dear Editor,}

It is now well known that somatic cells can be efficiently reprogrammed into induced pluripotent stem cells (iPSCs) by forced expression of defined factors [13]. These cells, like embryonic stem cells (ESCs), have true pluripotency as shown by the live, fertile mice that can be generated through the tetraploid complementation assay using these iPSCs $[4,5]$. So far, iPSCs have been generated from many species including mice, primate, rat, as well as pigs [1, 3, 6-12]; however, the latter failed to pass the gold standard test of pluripotency, that is, the tetraploid complementation assay, and iPSCs from some species have not even generated offspring with germline transmission. In addition, whether these iPSCs are capable of generating offspring through nuclear transfer remained to be determined.

In this study, we first looked at three iPSC lines generated using the four Yamanaka factors (Oct3/4, Sox2, Klf4, and c-Myc) [1] and tagged with an Oct4-fused enhanced green fluorescent protein (GFP) [13]. These iPSC lines were originated from B6D2 F1 mouse embryonic fibroblasts (MEF) isolated from E13.5 fetuses, as we previously reported [4]. They express the correct pluripotency markers, and can form teratomas in severe combined immunodeficient mice. All three lines have the ability to produce chimeric mice, but only one (IP14D-1) can generate live mice through tetraploid complementation.

To investigate the ability of different iPSC lines to generate cloned mice through somatic cell nuclear transfer (SCNT), and to compare with other nuclear donor cells, we first treated the three iPSC lines and their parental untransfected MEF cells, as well as two ESC lines (R1 and ESC2), with demecolcine to synchronize them at metaphase. Nuclei from each of the donor cell types were extracted and transferred to metaphase II (M II) oocytes of 8-week-old B6D2 F1 female mice using the Piezo-assisted one-step nuclear transfer method [14]. The reconstructed embryos were activated as previously described [15]. Embryos originated from iPSCs were selected by detection of fluorescence from Oct4-fused GFP expression. Interestingly, green fluorescence was observed starting from the eight-cell stage and was most apparent in the inner cell mass of the reconstructed embryos containing iPSC nuclei; this timing and localization coincide with normal Oct4 expression patterns. All of these embryos exhibited normal embryo morphology, indicating the likelihood of successful reprogramming of iPSCs by mouse M II ooplasm (Figure 1A-1F).

Preimplantation and postimplantation developmental efficiencies for the iPS-NT embryos were very similar to that of the ES-NT, and much higher than observed with MEF-NT embryos (Table 1). This difference is most exaggerated at the postimplantation stage. After transferring the nuclear transferred embryos back to the uterus of pseudopregnant white-coated CD-1 mice, 16 live cloned pups $(1.0 \%)$ were born after 19.5 days of gestation, 3 of which were derived from the two iPSC lines that were not tetraploid complementation compatible (Table 1 and Figure 1G). All of these 16 pups exhibited black coats, consistent with their origin from B6D2 F1 nuclear donor mice (Figure 1H). Similar efficiency was observed with ES-NT embryos when 20 cloned pups $(2.0 \%)$ were obtained. In contrast, neither live pups nor placentas developed from MEF-NT after 1333 MEF-reconstructed embryos were transferred into 34 pseudopregnant mice (Table 1). This result indicates that it is much easier to obtain cloned animals using MEF-derived iPSCs as donors than using the original untransfected MEF cells directly.

To confirm that the iPS-NT mice were generated by nuclear transfer from iPSCs reprogrammed using the Yamanaka factors, Southern blotting was performed using samples from iPS-NT mice and their parental iPSC lines. The identical viral integration patterns showed that the cloned mice were indeed generated from the corresponding iPSC lines (Figure 1J). Furthermore, to clearly identify the lineage of the iPS-NT mice, simple sequence length polymorphism (SSLP) analyses followed by PCR for various marker genes were performed. The placenta of iPS-NT mice had exactly the same marker profile as 

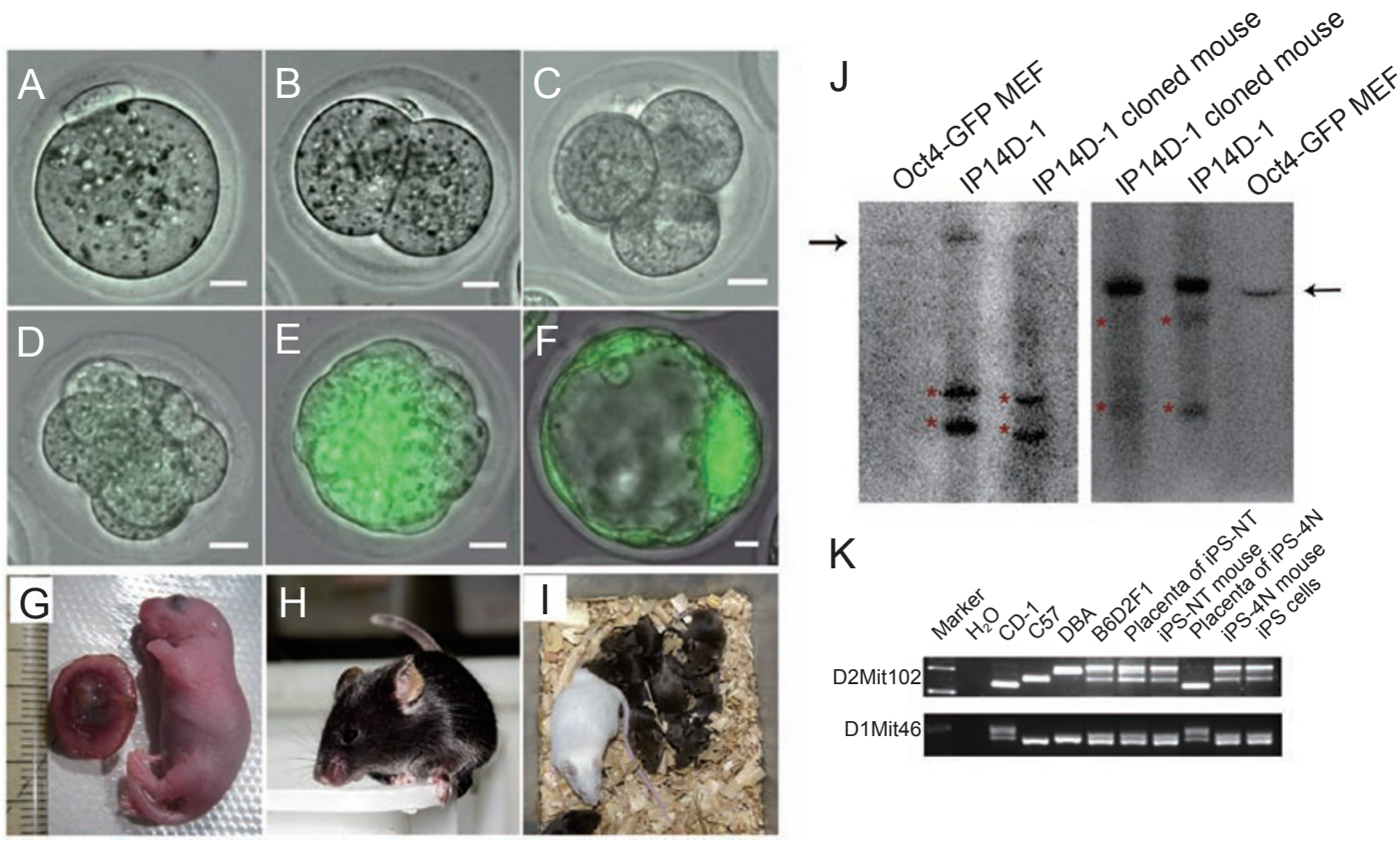

Figure 1 Derivation and characterization of iPSC-cloned mice (iPS-NT). (A-F) Preimplantation development of iPS-NT after nuclear transfer using Oct4-GFP iPSCs. Embryos at one-cell, two-cell, four-cell, eight-cell, morula and blastocyst stages are shown in A-F, respectively. The green fluorescence is observed starting at the eight-cell stage. The ruler bar represents 10 $\mu \mathrm{m}$. (G) An E19.5-cloned mouse derived from iPSC line, IP14D-1, by nuclear transfer. (H) An 8-week-old male iPS-NT mouse derived from the IP14D-1 line. The black coat color is consistent with the origin of the nuclear donor B6D2F1 mice. (I) The progeny of a male iPS-NT mouse mated with a female CD-1 mouse. The F1 pups shown here all have uniform brownish coats, typical for offspring from a mating of B6D2F1 (the origin of iPSC) and CD-1 mice. (J) Southern blot analysis showed identical viral integration patterns between the iPS-NT mice and their parental iPSC lines. Total genomic DNA was extracted from Oct4-GFP MEF, IP14D-1 and the corresponding NT mice. DNA samples digested with BamH1 and Bg/ll were hybridized with Oct4 and c-Myc cDNA probes, respectively. (K) SSLP analysis for lineage identification. The iPS-NT mouse and its placenta showed a polymorphic pattern similar to that of the iPSCs from B6D2F1 strain, and that different from the CD-1 strain and the placenta from the tetraploid-complemented iPS mice (iPS-4N). The digestion patterns of B6D2F1 parents (C57 and DBA) are also included.

that of their donor iPSC lines with B6D2F1 lineage, but the pattern was different from that of the pseudopregnant CD-1 mice that were recipients for embryo transfer. Note that the placentas of mice generated through tetraploid complementation using CD-1 blastocysts as tetraploid recipients would possess a marker profile similar to the CD-1 origin, instead (Figure $1 \mathrm{~K}$ ). These data clearly showed that the iPS-NT mice were generated by nuclear transfer using iPSCs as nuclear donors. To our knowledge, this is the first evidence showing that iPSCs can be cloned.

A total of 9 of 13 embryos (69.2\%) generated from the IP14D-1 line that was tetraploid complementation compatible survived to adult stage. They are healthy and have the same growth rate, life span, and behavior as wildtype mice. The eldest iPS-NT mouse is already 70 weeks (more than a year and 4 months) old. So far, no tumors were observed in these iPS-NT mice. To examine the fer- tility of our iPS-NT mice and the health of their progeny, a 7-week-old male iPS-NT mouse (black coated) was mated to a CD-1 female (white coated), and live-born and healthy progeny were obtained with brownish coats, typical for the offspring of black B6D2F1 crossed with white CD-1 mice (Figure 1I).

Somatic cell nuclear transfer has been widely used to study reprogramming. It is known that different types of nuclear donors may result in different success rates, with MEF being one of the more difficult types to clone, and its efficiency is variable across different mouse strains [2]. A few studies reported successful generation of cloned animals from fetal fibroblast cells $[16,17]$, but none had shown success using B6D2 F1 MEF as nuclear donors. In our study, we also observed different cloning efficiencies among different nuclear donors. Whereas full-term cloned mice using B6D2 F1 MEF were hard to acquire, we were able to generate full-term, viable and 


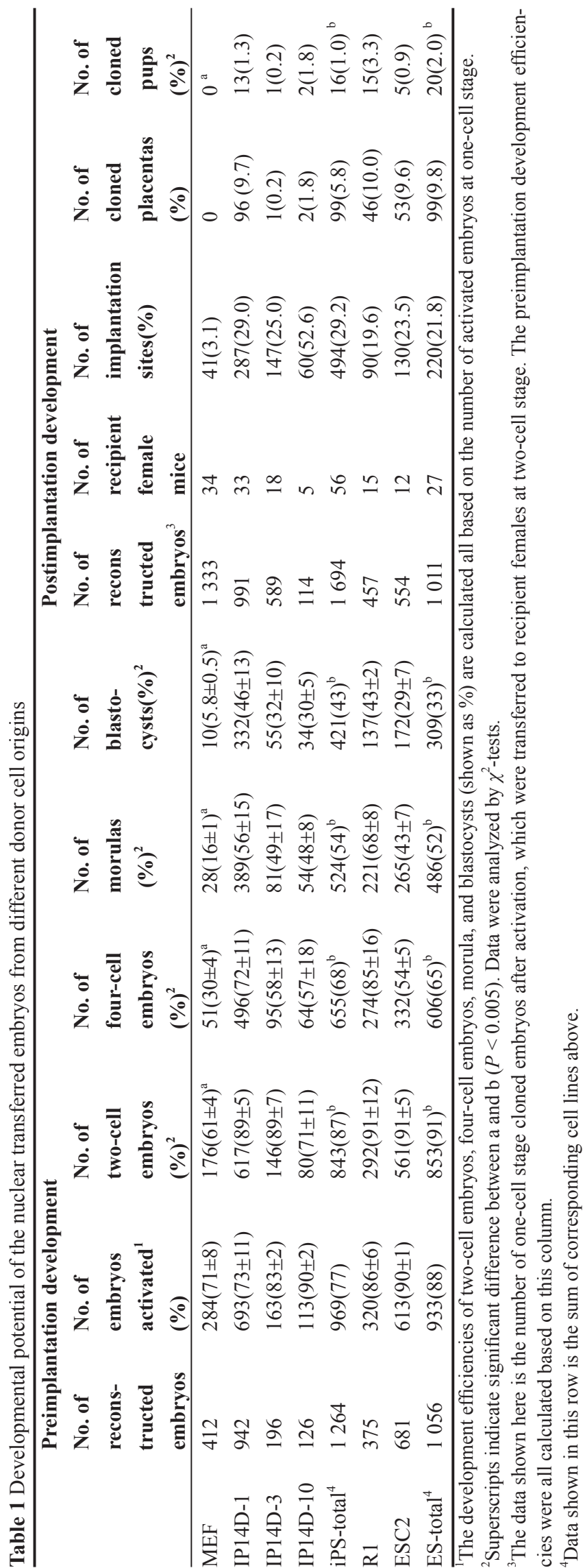

fertile cloned mice MEF-derived iPSCs from the same genetic background. Our work suggests that by using a combination of iPS and SCNT approaches, we are able to significantly improve the cloning efficiency of MEF cells. Whether the mechanisms of these two separate reprogramming methods are the same, and why different iPSC lines have different cloning efficiencies, remain interesting questions for further investigation.

In summary, we report for the first time, the generation of live mice using nuclear transfer from iPSCs originated from MEFs. This strategy not only shows that iPSCs have characteristics similar to ES cells, as determined here and previously, but also provides a new approach to obtain cloned animals from recalcitrant donor cells, which may facilitate the generation of transgenic animal models. The method also has an added advantage of generating genetically modified cloned animals engineered during the iPSC preparation process. Interestingly, live animals can be obtained from iPSCs that are not tetraploid complementation compatible. This presents a useful model for studying differences in the underlying mechanisms governing reprogramming through iPS and nuclear transfer technologies. iPSCs and their viable clones should offer potentially important utility for regenerative medicine, animal cloning, and cell development research.

\section{Acknowledgments}

This study was supported in part by grants from the National High Technology R \& D Program of China 2006AA02A101 and the China National Basic Research Program 2009CB941003. In addition, the authors would like to thank Eppendorf and Leica for supporting the laboratory.

Shuya Zhou ${ }^{1,2,{ }^{*}}$, Chenhui Ding ${ }^{1, *}$, Xiaoyang Zhao ${ }^{1,2, *}$, Eryao Wang ${ }^{1}$, Xiangpeng Dai ${ }^{1}$, Lei Liu ${ }^{1}$, Wei $\mathrm{Li}^{1,2}$, Zichuan Liu ${ }^{1,2}$, Haifeng Wan ${ }^{1,2}$, Chunjing Feng ${ }^{1,2}$, Tang Hai ${ }^{1}$, Liu Wang ${ }^{1}$, Qi Zhou ${ }^{1}$

${ }^{I}$ State Key Laboratory of Reproductive Biology, Institute of Zoology, Chinese Academy of Sciences, Beijing 100101, China; ${ }^{2}$ Graduate School of Chinese Academy of Sciences, Beijing 100049, China

*These three authors contributed equally to this work. Correspondence: Qi Zhou

${ }^{a}$ Tel: +86-10-64807299; Fax: +86-10-64807299

E-mail: qzhou@ioz.ac.cn

\section{References}

1 Takahashi K, Yamanaka S. Induction of pluripotent stem cells from mouse embryonic and adult fibroblast cultures by defined factors. Cell 2006; 126:663-676. 
2 Wakayama T. Production of cloned mice and ES cells from adult somatic cells by nuclear transfer: how to improve cloning efficiency? J Reprod Dev 2007; 53:13-26.

3 Yu J, Vodyanik MA, Smuga-Otto K, et al. Induced pluripotent stem cell lines derived from human somatic cells. Science 2007; 318:1917-1920.

4 Zhao XY, Li W, Lv Z, et al. iPS cells produce viable mice through tetraploid complementation. Nature 2009; 461:86-90.

5 Boland MJ, Hazen JL, Nazor KL, et al. Adult mice generated from induced pluripotent stem cells. Nature 2009; 461:91-4.

6 Esteban MA, Xu J, Yang J, et al. Generation of induced pluripotent stem cell lines from Tibetan miniature pig. J Biol Chem 2009; 284:17634-17640.

7 Ezashi T, Telugu BP, Alexenko AP, et al. Derivation of induced pluripotent stem cells from pig somatic cells. Proc Natl Acad Sci USA 2009; 106:10993-10998.

8 Li W, Wei W, Zhu S, et al. Generation of rat and human induced pluripotent stem cells by combining genetic reprogramming and chemical inhibitors. Cell Stem Cell 2009; 4:16-19.

9 Liao J, Cui C, Chen S, et al. Generation of induced pluripotent stem cell lines from adult rat cells. Cell Stem Cell 2009; 4:1115.

10 Liu H, Zhu F, Yong J, et al. Generation of induced pluripotent stem cells from adult rhesus monkey fibroblasts. Cell Stem
Cell 2008; 3:587-590.

11 Takahashi K, Tanabe K, Ohnuki M, et al. Induction of pluripotent stem cells from adult human fibroblasts by defined factors. Cell 2007; 131:861-872.

$12 \mathrm{Wu}$ Z, Chen J, Ren J, et al. Generation of pig induced pluripotent stem cells with a drug-inducible system. $\mathrm{J} \mathrm{Mol} \mathrm{Cell} \mathrm{Biol}$ 2009; 1:46-54.

13 Yoshimizu T, Sugiyama N, De Felice M, et al. Germlinespecific expression of the Oct-4/green fluorescent protein (GFP) transgene in mice. Dev Growth Differ 1999; 41:675-684.

14 Zhou Q, Jouneau A, Brochard V, Adenot P, Renard JP. Developmental potential of mouse embryos reconstructed from metaphase embryonic stem cell nuclei. Biol Reprod 2001; 65:412-419.

15 Wakayama T, Perry AC, Zuccotti M, Johnson KR, Yanagimachi R. Full-term development of mice from enucleated oocytes injected with cumulus cell nuclei. Nature 1998; 394:369374.

16 Ono Y, Shimozawa N, Ito M, Kono T. Cloned mice from fetal fibroblast cells arrested at metaphase by a serial nuclear transfer. Biol Reprod 2001; 64:44-50.

17 Saito M, Saga A, Matsuoka H. Production of a cloned mouse by nuclear transfer from a fetal fibroblast cell of a mouse closed colony strain. Exp Anim 2004; 53:467-469. 